\title{
La abstracción en Tomás de Aquino: una vía más allá de la epistemología tomista
}

\author{
Emiliano Cuccia y Ceferino Muñoz \\ UNCUyo/CONICET
}

\begin{abstract}
Resumen: El trabajo sugiere la necesidad de definir un nuevo enfoque bajo el cual estudiar la doctrina de la abstracción en Tomás de Aquino en orden a desentrañar el significado que le otorga en sus propios textos. Dicha tarea resulta relevante toda vez que los análisis realizados hasta la fecha a) muestran ciertas dificultades para interpretar correctamente algunos pasajes clave de la obra del Angélico, y b) han generado discusiones y controversias entre los especialistas a punto tal de impedir que se cuente en la actualidad con una doctrina común sobre el tema. En concreto, se analizan dos célebres textos del Aquinate para demostrar que los mismos no expresan lo que comúnmente se ha pretendido de ellos.
\end{abstract}

Palabras clave: abstracción; metafísica; gnoseología; epistemología

Abstract: "Abstraction in Thomas Aquinas: Beyond Thomistic Epistemology". This paper aims to justify the importance of a renewed way to analyze the doctrine of abstraction in Thomas Aquinas, considering that different studies so far a) show difficulties to correctly interpret some key texts of the Angelic, and b) have spawned debates and disagreements among scholars to the point of preventing the development of a common doctrine on the subject. Throughout the paper, two texts will be analyzed in order to show the contrast between what is said and what has been commonly interpreted from them.

Keywords: abstraction; metaphysics; gnoseology; epistemology 


\section{Consideraciones preliminares}

La doctrina de la abstracción ha sido uno de los tópicos más importantes dentro del tomismo. Prácticamente la totalidad de los pensadores adscriptos a esta corriente han interpretado la noción de abstracción como el término específico que designa el método según el cual el hombre conoce una realidad inteligible partiendo desde los datos sensibles, o bien como el modo según el cual la razón humana capta determinadas características de un objeto sin considerar otras. Esto implica que para estos autores la abstracción funciona como el elemento clave de la gnoseología de Tomás en la medida en que designa la operación por la cual el ser humano es capaz de obtener los objetos de conocimiento intelectual a partir del análisis de los objetos materiales. Paralelamente, lo abstracto ha significado el producto final de dicho proceso mental.

En esta línea existe una lectura del término que podría ser denominada epistemológica, en tanto considera la presencia de una operación abstractiva, específica del intelecto pasivo, que funcionaría como la causa que fundamenta la distinción entre los objetos científicos y que, consecuentemente, justifica la certeza y objetividad de la ciencia. Ahora bien, es posible identificar algunos elementos que -al menos en un primer momento- generan ciertas dudas acerca de la precisión de tal interpretación con respecto a la letra del Aquinate. Por un lado, pueden encontrarse pasajes dentro de las obras de Tomás -y en contextos donde el tema discutido toca de lleno la cuestión de los objetos de la ciencia- cuya plena comprensión se hace imposible si se considera al término abstracción solo como un modo de designar un proceso u operación intelectual. Por otro lado, también puede comprobarse que aquellos pasajes en los cuales los comentadores pretenden apoyarse para justificar sus interpretaciones y desarrollos no siempre expresan la doctrina que estos últimos afirman.

Esto último no representaría en sí mismo un inconveniente en tanto es 246 esperable que toda tradición filosófica fundada en torno al pensamiento de un autor de la talla del Aquinate intente extender los límites de sus conclusiones más allá de lo que se encuentra expresamente escrito. Sin embargo, debe notarse que, en este caso concreto, lo que ha motivado tales lecturas es un afán muy particular: responder con los textos del monje medieval a la pregunta moderna por la propia posibilidad y la certeza del conocimiento científico del 
mundo ${ }^{1}$. Se trata de una cuestión que trasciende claramente su horizonte hermenéutico y que -de no hacerse las aclaraciones pertinentes- pueden dejarlo expuesto a fuertes objeciones frente a las cuales poca defensa podría hallarse en sus textos ${ }^{2}$. De este modo, aunque los autores que defienden la interpretación de la operación abstractiva entienden que mediante ella se asegura la defensa del realismo gnoseológico tomasiano, la misma ha propiciado también el surgimiento de objetores para quienes hablar del conocimiento abstractivo en tales términos implica admitir una mediación dentro del acto cognoscitivo merced a la cual no sería estrictamente la realidad lo que se conoce sino una representación de la misma generada por el propio intelecto ${ }^{3}$. A su vez, esto ha generado reacciones de quienes, conscientes de la falacia en la que cae cualquier propuesta representacionalista, intentan demostrar que la repraesentatio a la

\footnotetext{
1 Es célebre en este sentido la exégesis de Jacques Maritain en su libro Les degres du savoir donde la abstracción es propuesta como el medio por el cual la inteligencia obtiene sus objetos a partir de las cosas materiales y contingentes (Cf. Maritain, J., Los grados del saber. Distinguir para unir, Buenos Aires: Club de Lectores, 1978, pp. 53-54). Sin embargo, también es posible encontrar lecturas similares en otros autores como Suzanne Mansion ("L' intelligibilité metaphysique d'apres le 'Proemium' du Commentaire de Saint Thomas a la 'Metaphysique' d'Aristote", en: Rivista di Filosofia Neo-Scolastica, 70 (1978), pp. 49-62, p. 52), Leo Elders (Faith and Science: an Introduction to St. Thomas' Expositio in Boethii De Trinitate, Studia Universitatis S. Thomae in Urbe, Roma: Herder, 1974, p. 99), Philip Merlan (From Platonism to Neoplatonism, v. III, La Haya: Martinus Nijhoff, 1975, pp. 77-81), Armand Maurer (The Division and Methods of the Sciences, 1986, pp. xvi-xvii), K. Nordberg ('Abstraction and Separation in the Light of the Historical Roots of Thomas' Tripartition of the Theoretical Sciences", en: Knowledge and the Sciences in Medieval Philosophy. Proceedings of the Eighth International Congress of Medieval Philosophy (1987), Helsinski: Yliopistopaino, 1990, pp. 144-159), Joseph Kockelmans (The Metaphysics of Aquinas: a Systematic Presentation, Lovaina: Bibliotheek van de Faculteit Godgeleerdheid, 2001, p. 40), Lorenzo Vicente Burgoa ("La abstracción formal y la validación del razonamiento inductivo", en: Sapientia, 62 (2007), pp. 129-178, p. 133) y Ariberto Acerbi (“Aquinas's Commentary on Boethius' De Trinitate", en: Review of Metaphysics, LXVI, 2 (2012), pp. 317-338, p. 319), entre muchos otros.

${ }_{2}$ Y muy posiblemente esto sea así en tanto se trata de problemas que históricamente surgieron después de la muerte del monje. Tal es el caso también de la pretensión de algunos autores por justificar la distinción entre la fisica empírica y la filosofia de la naturaleza desde la propia operación abstractiva planteada por el Aquinate. Distinción que, claro está, no tiene razón de ser en la mente del Aquinate ni en la de sus contemporáneos. Tanto Fergus Kerr como Alasdair MacIntyre han advertido sobre el error de poner a Tomás a discutir tales cuestiones planteadas en un contexto muy diferente al suyo propio (cf. MacIntyre, A.C., First Principles, Final Ends, and Contemporary Philosophical Issues, The Aquinas Lecture, Milwaukee: Marquette University Press, 1990, y Kerr, F., “¿Un tomismo analitico?”, en: Civilizar Ciencias Sociales y Humanas, 12 (2012), pp. 149-158. Ver también Bonino, S-T., "Le thomisme de 1962 à 2012. Vue panoramique", en: Nova et Vetera, 87 (2012), pp. 419-446.

3 Un caso paradigmático lo constituye Robert Pasnau, quien afirma que, a pesar de la insistencia del propio Aquinate en que el objeto del conocimiento son las propias quididades de las cosas, su defensa del conocimiento por similitudes originadas a partir de la abstracción desde los fantasmas se encolumna más en la línea de un representacionismo que de un realismo ( $C f$. Pasnau, R., Theories of Cognition in the Later Middle Ages, Cambridge: Cambridge University Press, 1997, p. 197).
} 
que se refiere Tomás en varios pasajes no es asimilable a lo que se ha entendido modernamente por tal concepto ${ }^{4}$.

Frente a este estado de cosas, consideramos relevante llamar la atención sobre la posibilidad de que el auténtico sentido que encierra la abstracción para el Aquinate no se condiga de un modo pleno con las interpretaciones que se han hecho en siglos posteriores ${ }^{5}$. En dicha linea, el objetivo del presente trabajo consistirá en analizar dos textos que, a nuestro modo de ver, pueden servir como casos testigo en tanto son considerados por un buen número de autores como los sitios clave donde Tomás desarrolla una doctrina de la abstracción en sentido epistemológico, esto es, como causa de la obtención de los objetos de conocimiento y de la división de las ciencias especulativas ${ }^{6}$. Con todo, el análisis pondrá de manifiesto que lo afirmado allí por el Aquinate dista de las pretensiones de sus comentadores.

Lo que se buscará, en definitiva, es mostrar de qué modo una relectura de estos textos posibilita una comprensión más rica, capaz de superar algunas de las dificultades hermenéuticas que surgen a propósito de una lectura epistemológica de los textos. Previamente, y con el objeto de ganar en claridad, consideramos metodológicamente relevante realizar una breve síntesis que exponga con mayor amplitud qué es lo que fundamentalmente se entiende cuando se habla de abstracción dentro del ámbito general del tomismo.

4 Cf. Moya Cañas, P., "Inmanencia, intencionalidad y representación en Tomás de Aquino", en: Veritas: Revista de Filosofia, 28 (2013), pp. 113-131.

5 Un aporte interesante y reciente a esta misma tarea lo constituyen los estudios publicados por Therese Scarpelli Cory, quien ha demostrado de qué modo la abstracción iluminativa del intelecto agente ha sido erróneamente interpretada como un proceso de selección o de sustracción de elementos universales. La autora recurre a los textos del Aquinate y al análisis de las influencias recibidas desde los filósofos islámicos para indicar que, en realidad, tal abstracción señala un proceso co-causal mediante el cual el intelecto agente le presta su fuerza intelectiva al fantasma imaginativo para que este actúe su semejanza en el pasivo ( $C f$. Cory, T.S., "Averroes and Aquinas on the Agent Intellect's Causation of the Intelligible", en: Recherches de Théologie et Philosophie Médiévales, v. LXXXII, 1 (2015), pp. 1-60, pp. 36 y 53). Sin embargo, por razones que quedarán expuestas más adelante, creemos que el aporte de Cory es limitado en tanto sigue considerando la necesidad de una abstracción adicional a la del agente que permita distinguir y determinar los objetos científicos mediante un proceso de selección ulterior. Para la autora, tal abstracción sería la que se describe en Super Boethium De Trinitate q. 5, a. 3 (Cf. Cory, T.S., "Rethinking Abstractionism: Aquinas's Intellectual Light and Some Arabic Sources", en: Journal of the History of Philosophy, v. LIII, 4 (2015), pp. 607-646, p. 630).

6 También es posible emprender otro camino con vistas al mismo objetivo. Este consistiría en analizar textos donde la abstracción es puesta en relación directa con la división de las ciencias especulativas y sus objetos, pero sin que con tal término se esté haciendo referencia a una operación intelectual. Con todo, las limitaciones de espacio exigen que tal análisis se encare en un trabajo especialmente dedicado. 


\section{La abstracción en el contexto de la tradición tomista}

Tal vez el antecedente más antiguo y a la vez relevante de una interpretación de la doctrina de la abstracción tomasiana se encuentra en los escritos de su célebre comentador Tomás de Vio. Todo apunta a que el Cardenal es el creador de la clasificación de la abstracción tomasiana en neutra, total y formal, enumerando a su vez para esta última tres tipos o grados que se corresponderían con el método de cada una de las ciencias especulativas. Esta distinción es introducida y desarrollada en sus comentarios al proemio del De ente et essentia, donde busca explicar de qué modo puede entenderse que el ente sea lo primero conocido y, a la vez, el objeto propio de la más alta ciencia especulativa: la metafísica 7 . Su respuesta se centra en afirmar que "el ente puede coronar el acto del intelecto de un triple modo"8; lo cual no implica más que admitir que el ente se constituye como objeto de conocimiento diferentemente según los tres tipos generales de abstracción citados más arriba: según la abstracción neutra, el ente es constituido como lo primero conocido de un modo confuso; según el proceso de abstracción total, como un objeto meramente lógico; y según el proceso de la triple abstracción formal, como objeto de la ciencia física (ente material), matemática (ente quantum) o metafisica (ente en cuanto ente), respectivamente.

Más allá de las particularidades que hacen a la explicación de Cayetano, lo que resulta interesante destacar es el rol que se le atribuye a la operación abstractiva como modo de explicar la obtención de la multiplicidad de objetos que perfeccionan la inteligencia. En este sentido es clave la distinción entre abstractio totalis y abstractio formalis como dos operaciones del intelecto pasivo ${ }^{9}$ que permiten acceder a sendos planos distintos del conocimiento: el de la lógica y el de la ciencia especulativa, respectivamente. Esta idea fue continuada y profundizada por numerosos autores posteriores ${ }^{10}$ y de una forma particular en los albores del siglo XX, a punto tal de conformar una doctrina clásica acerca

\footnotetext{
7 Tal cuestión está claramente motivada por las objeciones del escotista Antonio Trombetta, contemporáneo del Cardenal de Gaeta ( $C f$. Muñoz Medina, C., "Notas sobre el primum cognitum en Cayetano y su vínculo con la metafisica", en: Anales del seminario de Historia de la Filosofía, 32 (2015), pp. 127-142, pp. 130-131).

8 In de Ente et Essentia, p. 221.

9 La distinción entre la abstracción del intelecto agente y la del pasivo se encuentra en el comentario de Cayetano a la cuestión 85 de la primera parte de la Summa Theologiae (Cf. Aquinatis, T., Pars prima Summae theologiae. "Commentaria Cardenalis Caietani", t. V, p. 323).

${ }^{10}$ Tal vez uno de los continuadores más célebres es Juan de Santo Tomás. Cf. I. a. S. Thoma. Cursus Philosophicus Thomisticus, Ars Logica, II, q. 5, p. 128 y q. 27, pp. 327-332.
} 
del concepto tomasino de abstracción ${ }^{11}$ caracterizada por dos notas: 1) admitir que la doctrina acerca de la abstracción desarrollada en los comentarios de Cayetano coincidía -al menos en lo conceptual- con el pensamiento del Doctor Angélico, y 2) considerar el término abstracción en un sentido principalmente epistemológico, es decir, como la respuesta que podía darse desde el pensamiento tomista a la siguiente pregunta: ¿cómo puede el hombre garantizar la certeza del conocimiento universal y necesario, partiendo de lo particular y contingente? ${ }^{12}$. Sin embargo, a partir del descubrimiento, en la década del 40, de una edición autógrafa del comentario de Tomás de Aquino al De Trinitate de Boecio, la supuesta coincidencia doctrinal entre el Doctor Común y sus comentadores inmediatos fue puesta en duda. En efecto, el estudio de dicho hallazgo permitió a autores como Geiger ${ }^{13}$ y Merlan ${ }^{14}$ plantear la existencia de fuertes diferencias entre la doctrina tomista clásica acerca de la abstracción y lo que el propio Aquinate parecía afirmar, especialmente en lo referente a la relación de la abstracción con la determinación del objeto de la metafísica. A partir de estas consideraciones, otros autores suscribieron una reducción del ámbito de

\footnotetext{
${ }^{11}$ Entre los más importantes representantes de dicha tradición puede citarse a Ramírez, S.M., De ipsa philosophia in universum, Madrid: Instituto de Filosofia "Luis Vives", 1970, pp. 70-74; Maritain, J., Distinguer pour unir, París: Desclée, 1932; Leroy, M-V., "Le savoir speculatif", en: Revue Thomiste: Revue doctrinale de Theologie et de Philosophie v. XLVIII, 1-2 (1948), pp. 236-339; Simmons, E.D., "The Thomistic Doctrine of the Three Degrees of Formal Abstraction", en: Thomist: A Speculative Quarterly Review, 22 (1959), pp. 37-67; Ferrari, L.C., "A Thomistic Appraisal of Thomas de Vio Cajetan's 'Abstractio Totalis"', en: Angelicum: Periodicum Trimestre Pontificiae Studiorum Universitatis e Sancto Thoma Aquinate in Urbe, 42 (1965), pp. 441-462; López Salgado, C., "Abstractio y separatio como acceso a la metafisica", en: Sapientia, v. XIX, 72 (1964), pp. 102-116; Ponferrada, G.E., "Nota sobre los 'grados de la abstracción", en: Sapientia, 33 (1978), pp. 267-284; Llano, C., "La 'Introducción' a la Crítica de la Razón Pura y el Comentario al De Trinitate de Boecio de Santo Tomás de Aquino", en: Tópicos. Revista de Filosofía, 21 (2001), pp. 27-67; Burgoa, V., “¿Abstracción formal o separación? Desde Tomás de Aquino a Cayetano", en: Estudios Filosóficos, 138 (1999), pp. 211-256.

${ }^{12}$ Esta pregunta aparece formulada de manera casi textual por Jacques Maritain en su libro Distinguer pour unir, donde la abstracción es propuesta como el medio por el cual la inteligencia asegura la obtención de sus objetos universales y necesarios a partir de las cosas materiales y contingentes ( $C f$. la versión en castellano Maritain, J., Los grados del saber. Distinguir para unir, 250 1978, pp. 53-54). También es posible encontrar formulaciones equivalentes en los otros autores citados. Dicho sea de paso, este modo de encarar el tema de la abstracción parece implicar una innovación con respecto a lo que Cayetano y Juan de Santo Tomás interpretaron, ya que no hay indicios de que estos últimos hayan tenido la intención de plantear el asunto desde la óptica de un intento por justificar la posibilidad del conocimiento intelectual de lo extramental.

${ }^{13}$ Geiger, L.B., "Abstraction et Séparation d' après Saint Thomas In de Trinitate, Q. 5, A. 3", en: Ruedi, I. (ed.), Penser avec Thomas d' Aquin, Friburgo/Paris: Éditions Universitaires-Éditions du Cerf, 2000, pp. 139-1832000.

${ }^{14}$ Merlan, P., "Abstraction and Metaphysics in St. Thomas' Summa", en: Journal of the History of Ideas, 14 (1953), pp. 284-291 y From Platonism to Neoplatonism, 1975, pp. 69-70.
} 
la abstracción al plano de las ciencias natural y matemática, reservando para la metafísica una operación intelectual distinta: la separatio ${ }^{15}$.

Sin embargo, incluso con todas las precisiones ganadas a raíz de estos estudios, la interpretación del término abstracción, tal como aparece utilizado en las obras de Tomás de Aquino, continuó generando perplejidades debido principalmente a que el mismo no parece ser tomado en todo momento con la propiedad que sería lógico reclamarle a un elemento tan importante de su corpus doctrinal. En efecto, lo que ha llamado la atención de varios autores ${ }^{16}$ es la aparente discrecionalidad con la que Tomás llama abstractos a ciertos objetos que otras veces denomina separados, incluso en un mismo texto ${ }^{17}$, o que atribuya un mismo modo de abstracción a dos tipos de objetos pertenecientes a ciencias completamente distintas ${ }^{18}$. Esto constituye un gran problema si se pretende que la abstractio designe un determinado proceso intelectual que permita el acceso a un ámbito particular y preciso del conocimiento conformando un objeto específico.

${ }^{15}$ Entre ellos puede contarse a Nordberg, K., "Abstraction and Separation in the Light of the Historical Roots of Thomas' Tripartition of the Theoretical Sciences", en: Tyorinoja, R. (ed.), Knowledge and the Sciences in Medieval Philosophy. Proceedings of the Eighth International Congress of Medieval Philosophy (1987), Helsinski: Ylipostipaino, 1990, pp. 144-159; Kockelmans, J.J., The Metaphysics of Aquinas: a Systematic Presentation, Lovaina: Bibliotheek van de Faculteit Godgeleerdheid, 2001, especialmente pp. 39-40, y, más recientemente, Pascual Aguirre, R., La división de las ciencias especulativas en Santo Tomás de Aquino. Excerpta ex dissertatione ad Doctorandum in Facultate Philosophiae, Pontificia Universitas Gregoriana, 2003, p. 95; Nuñez Pliego, J.M., "Abstracción y separación. Estudio sobre la metafísica de Tomás de Aquino", en: Cuadernos de Anuario Filosófico, González Eunsa, A. (ed.), Pamplona, 2008, pp. 30-37 y Acerbi, A., Aquinas's Commentary on Boethius' De Trinitate, en: Review of Metaphysics, v. LXVI, 2 (2012), pp. 317-338. ${ }^{16}$ Cf. Burgoa, V., "¿Abstracción formal o separación? Desde Tomás de Aquino a Cayetano", p. 247; Pascual Aguirre, R., La división de las ciencias especulativas en Santo Tomás de Aquino, p. 29, pero también ya lo insinuaban Leroy, M-V. (Le savoir speculatif, p. 333) y Edward Simmons (“The Thomistic Doctrine of the Three Degrees of Formal Abstraction", pp. 43-46).

${ }_{17}$ Un caso paradigmático lo constituye la cuestión quinta del Super Boethium De Trinitate queen su artículo $1^{\circ}$ habla de una triple separación como causa de la distinción de los objetos, pero que en el artículo $3^{\circ}$ introduce la célebre distinción entre dos operaciones abstractivas y una separativa. Frente a esta aparente contradicción se han esbozado algunas posibles soluciones como atribuirla a una maduración del tema por parte del propio Aquinate ( $C f$. Pascual Aguirre, R., La división de las ciencias especulativas en Santo Tomás de Aquino, p. 29), una concesión al lenguaje utilizado por Boecio (Cf. Maurer, A.A., The Division and Methods of the Sciences, 1986, pp. xvi-xvii) o simplemente un uso laxo de los términos (Cf. Elders, L., Faith and Science: an Introduction to St. Thomas' Expositio in Boethii De Trinitate, pp. 91-105). Claro está que estas soluciones solo tienen sentido en el caso de que la supuesta contradicción resulte real, lo cual implicaría admitir desde ya que Tomás introduce la triple operación distintiva del artículo tercero como fundamento causal de la división de la ciencia. Este es precisamente una de los puntos que se intentará discutir en lo que sigue.

${ }_{18}$ Por ejemplo, esto ocurre en el comentario a la Física aristotélica cuando atribuye la abstractio universalis a particulari (también conocida como totius) tanto a los objetos de la física como de la lógica (Cf. In Physica II, lectio 3, n. 5, p. 63). 
En otras palabras, a partir del descubrimiento del texto autógrafo del comentario al De Trinitate y de los estudios realizados acerca de la separatio, quedó en evidencia para muchos autores que Cayetano no afirma exactamente lo mismo que Tomás de Aquino, al menos acerca de la abstracción. A pesar de ello, a nuestro modo de ver, esto no implica que se haya podido probar de un modo definitivo qué intenta decir exactamente el Aquinate al hablar de abstracción, en tanto muchas de las cosas que afirman sobre ella no parecen encontrar un pleno correlato en sus textos. Es aquí donde se fundamenta el planteo basal de este trabajo: que la interpretación de los textos sobre la abstracción -incluso en aquellos autores que innovan sobre la doctrina más tradicional- sigue siendo inapropiada en tanto busca en ella una respuesta para una pregunta que trasciende el universo conceptual de Tomás. No solo se pretende que la misma explique cómo conocemos la realidad sino también que dé cuenta del mismo objeto de conocimiento. Es decir, que justifique el conocimiento como tal, sea del objeto en sentido llano (gnoseología) o sea del objeto de cada ciencia o género de ciencia en particular (epistemología) ${ }^{19}$.

Como se indicó más arriba, un camino que permite poner en evidencia dicha inadecuación entre la fuente y los comentaristas consiste en mostrar que aquellos textos del Aquinate en los cuales los segundos se apoyan para fundamentar sus interpretaciones desarrollan una doctrina bastante diversa de lo pretendido. En lo que resta del trabajo se emprenderá este camino con el objetivo final de dejar planteada la necesidad de encarar un estudio de la abstracción en Tomás bajo un nuevo enfoque que haga un paréntesis sobre las motivaciones que han caracterizado los análisis de los últimos años.

\section{El análisis de las fuentes consideradas por los comentaristas}

Son numerosos los textos de Tomás de Aquino a los que sus comentadores acuden para justificar sus propios desarrollos teóricos acerca de la abstracción científica ${ }^{20}$. Con todo, existen dos que han recibido una atención especial en tanto han sido considerados como pasajes paralelos que describirían la supuesta doctrina tomística de la obtención de los objetos científicos

\footnotetext{
${ }^{19}$ Un estudio aproximativo que plantea el modo en que ciertas concesiones modernas de la epistemologia tomista la alejan del planteo original de la scientia tomasiana puede encontrarse en Mendoza, J.M.F., "La epistemología tomista: un designio de la originaria noción tomística de ciencia", en: Ciencia Tomista, v. CXL, 452 (2013), pp. 389-416.

${ }^{20}$ Entre otros, puede citarse a In Metaphisic., Proemium; In Physic. II, 3; In De Anima III, 8, In De sensu et sensato I, 1.
} 
mediante distinciones intelectuales: Super Boethium De Trinitate, q. 5, a. 1 y Summa Theologiae I, q. 85, a. 1, ad. 2. Tal ha sido su impacto que incluso las diferencias terminológicas evidentes que existen entre ellos son las que han alimentado gran parte de las discusiones sobre la abstracción de los últimos cincuenta años. Por ello, y en razón del carácter paradigmático que poseen, consideramos interesante centrar el análisis en ellos.

\subsection{Super Boethium De Trinitate, q. 5, a. 1, co}

En la obra mencionada, Santo Tomás se enfrenta a los problemas que implica dividir la ciencia especulativa en tres partes teniendo en cuenta que, si es correcta la afirmación de Aristóteles expuesta en su De Anima acerca de que la ciencia especulativa es acerca de las ousias y de que hay tantas ciencias cuantas ousias hayan, parecería que la división tripartita es errónea. Esto podría concluirse en tanto se piensa que las cosas se dividen en dos géneros supremos, a saber: materiales y separadas ${ }^{21}$. Asi, debería haber solo dos géneros de ciencia especulativa y no tres. Sin embargo, es el propio Estagirita el que establece en el libro VI de su Metafísica la división en física, matemática y ciencia primera o metafísica ${ }^{22}$.

En orden a resolver esta dificultad, el Doctor Angélico afirma que "cuando los hábitos o las potencias se diferencian según los objetos, no se diferencian según cualesquiera diferencias de los objetos sino según aquellas que son propiamente de los objetos en cuanto son objetos" 23 . De igual modo, "es conveniente distinguir las ciencias especulativas a través de las diferencias de los especulables en cuanto son especulables" ${ }^{24}$. A renglón seguido establece que lo que caracteriza al especulable como tal es la remoción de la materia y el movimiento, pero, como se dan distintos grados en esta remoción, hay entonces diversos tipos de especulables y diversas ciencias especulativas que los estudian.

${ }^{21}$ Cf. Super De Trinitate q. 5, a. 1, arg. 8.

${ }^{22}$ Cf. Metafísica, 1026a18, y Super De Trinitate, q. 5, a. 1, sc 1. Se ha discutido si esta tripartición y la correspondiente referencia al orden de las ousías como causas fundamentativas constituye la opinión final del estagirita sobre el tema o si se trata más bien de un resabio platónico que luego fue superado (Cf. Merlan, P., From Platonism to Neoplatonism, La Haya: Martinus Nijhoff, 1975, pp. 59-87). Con todo, consideramos que esta discusión no afecta en lo sustancial lo que aquí se discute toda vez que a) Tomás parece considerar como correcto tal criterio de división, y b) la tradición tomista considera que, con ocasión de tal tripartición, el Aquinate desarrollaría un criterio fundamentativo de la misma que resultaría superador en tanto estaría basado en el recurso a las operaciones intelectuales. Esto último es lo que se pretende cuestionar en el presente escrito.

${ }^{23}$ Super De Trinitate, q. 5, a. 1, co., p. 138.

24 Ibid. 
Todo lo dicho hasta aquí, en tanto constituye una mera presentación de las afirmaciones del Aquinate, no reviste mayores inconvenientes para ser interpretado en los términos de aquellos autores que se han abocado al estudio de este texto desde la óptica epistemológica ya explicada. El denominador común en todos ellos reside en considerar que esta remoción de la materia de la que se habla supone una operación intelectual abstractiva que toma lo formal y descarta lo material de tres modos distintos. Así, la fundamentación y división de las ciencias para Tomás se apoyaría causalmente en las operaciones que el intelecto humano realiza para conformar el objeto de conocimiento. Sin embargo, creemos que un análisis detenido del texto es capaz de mostrar una realidad un tanto diferente. Debe notarse que en ningún momento el monje habla aquí de una operación intelectual, ni mucho menos de alguna a la que se le atribuya el mentado rol ${ }^{25}$. Según sus propias palabras, el núcleo de la distinción lo constituye la separación que los mismos objetos especulables tienen frente a la materia, la cual debe entenderse en términos de independencia. Como dicha independencia puede ser según el modo de existencia o según el modo de ser entendida, es posible enumerar tres tipos de relación entre los objetos y la materia.

Así, los objetos sobre los que especula la metafísica son independientes de la materia según su modo de existencia ya que o bien no existen en la materia o bien pueden no hacerlo. Esto implica que se encuentran en un nivel más alto de inteligibilidad con respecto a los demás objetos. Estos últimos necesitan forzosamente de la materia para existir, pero entre ellos existe una distinción, porque los hay que, aunque no puedan existir fuera de la materia, sí pueden ser entendidos sin ella ya que la misma no entra en su definición: estos son los especulables matemáticos como la línea y el punto. No obstante, hay otros que no solo existen en la materia, sino que la misma es parte de su definición y deben ser entendidos con ella ${ }^{26}$ : son los especulables físicos como el hombre o el asno.

Pasando en limpio lo visto hasta aquí, resulta necesario admitir que cuando se habla de la independencia existencial con respecto a la materia no se está reclamando la intervención de ninguna operación intelectual que produzca

\footnotetext{
${ }^{25}$ En sentido estricto, ni siquiera utiliza aquí los términos abstraer o abstracción. Aunque sí habla de separatio a materia y de un ordo remotionis, no parece que ninguno de estos términos haga referencia a una operación del intelecto, como se verá a continuación.

${ }^{26}$ Como explicará en el artículo $2^{\circ}$, la materia que forma parte de la definición de los especulables físicos es la materia sensible no signada, esto es, la materia sin las determinaciones particulares que la convierten en principio de individuación ( $C f$. Super De Trinitate, q. 5, a. 2, co.).
} 
esta distinción, sino que la misma se plantea como algo ya dado. Ciertamente, hay cosas que no necesitan de la materia para existir o que incluso la excluyen necesariamente. Tales entes son inteligibles por sí mismos y su remoción de la materia no es fruto de una operación intelectual, sino consecuencia del estatus ontológico de la propia realidad estudiada.

Pero, ¿qué ocurre en el caso de aquellos entes que solo existen en la materia? ¿Acaso no se justificaría aquí la necesidad de una operación abstractiva que separe o remueva lo formal de lo material para causar la distinción entre los especulables? ¿No se refiere a eso cuando dice que la separación es según el intelecto? En mi opinión, no hay evidencia que lleve a concluir necesariamente tal cosa. Más bien existen elementos que llevan a pensar que, cuando se habla de independencia o separación según el intelecto, lo que se indica es algo que implica un rol más bien pasivo para este último.

Efectivamente, en el mismo corpus del artículo primero afirma que depender de la materia según el intelecto se identifica con no poder ser definido sin hacer referencia a la materia. Puede que lo dicho -considerado en solitario- no aporte mucho a la causa, pero si se trae conjuntamente a cuento lo que se expresa sobre la definición en un texto cronológicamente cercano a este el panorama cambia completamente. En el capítulo primero del De ente et essentia, Tomás indica que el rol de la definición es señalar lo que la cosa es, su quididad, que no es más que otro nombre para designar la esencia ${ }^{27}$. Es posible hallar la misma doctrina en un texto de madurez como el Comentario a la Metafísica, más precisamente en el libro VIII28. De este modo, si un elemento, principio o característica no puede ser omitido en la definición de un ente es porque se trata de un componente fundamental de la esencia y -contrariamente- si puede ser omitido es porque no lo es.

Visto esto, la separatio secundum intellectum del especulable con respecto a la materia, tal como es introducida en el Super Boethium De Trinitate, no sería más que un modo de referirse a la distinción que la propia esencia presenta con respecto a aquellos elementos que, aunque la acompañen necesariamente en su existencia aquí y ahora, no le pertenecen con propiedad. Por lo demás, resulta claro que tal distinción no es fruto o consecuencia de la operación intelectual. Más bien corresponde decir que es la inteligencia al concebir la que no puede desconocerla. En la medida en que, según Tomás, una cosa es inteligible en tanto es un acto (como las sustancias separadas), posee un acto (como los entes

${ }^{27}$ Cf. De ente et essentia, cap. 1, p. 369.

28 In Metaphysic. VIII, lectio $1, \S 1710$. 
compuestos) o se relaciona con un acto (como la materia prima con respecto a la forma ${ }^{29}$ ), la inteligencia está obligada a conocer cada cosa con todo aquello que su naturaleza exige de suyo. Pero, por lo mismo, la entiende librada a su vez de todas aquellas otras que no pertenecen a su esencia.

Así, es razonable concluir aquí -con base en la evidencia aportada- que con el término especulable el Aquinate no está haciendo referencia al objeto intelectual como si se tratase del producto de una distinción causada por la operación abstractiva de la inteligencia humana. Se refiere, más bien, a la cosa misma, al ente que es inteligible en tanto es o posee una determinada esencia existiendo en acto. Por lo mismo, la separación del especulable con respecto a la materia no indica otra cosa que el modo de independencia de tal o cual ente -sea este substancial o accidental- frente a la materia sensible. Tal independencia puede darse en el plano existencial, como es el caso de las sustancias simples, o solo en el de la composición esencial, como acaece con los entes matemáticos.

Podría objetarse que lo que aquí se afirma es desmentido por la introducción, en el artículo $3^{\circ}$ de la cuestión quinta, del triple modo de distinguir del intelecto (según su primera o segunda operación) y su vinculación con las tres ciencias especulativas. Alli se enumeran dos operaciones abstractivas (universalis a particulari y formae a materia) junto a una separativa que competen respectivamente a la fisica, la matemática y la metafisica ${ }^{30}$. Ciertamente, resulta comprensible que este pasaje haya dado pie a la interpretación epistemológica de la abstracción, máxime cuando el mismo es leído bajo la convicción de que Tomás de Aquino reclama la postulación de operaciones intelectuales diferentes para la obtención de diversos objetos cognitivos. Es por ello que el artículo $3^{\circ}$ ha funcionado comúnmente como el gozne hermenéutico en torno al cual es interpretada toda la cuestión quinta del Super De Trinitate ${ }^{31}$.

No obstante, consideramos que tal deducción no es algo que deba imponerse sin más, ya que el rol de las distinciones intelectuales podría ser interpretado de un modo diferente. Incluso, este último resultaría más compatible con lo afirmado en el artículo $1^{\circ}$ en tanto mostraría a la distinción de operaciones

29 Cf. Super De Trinitate, q. 5, a. 3, lin. 121-130, p. 147.

${ }^{30}$ Cf. Super De Trinitate, q. 5, a. 3, co., lin. 275-285, p. 149.

31 Esto es lo que causa que la triple separación formulada en el artículo $1^{\circ}$ sea vista o bien como una mera concesión lingüística o bien como una concepción superada inmediatamente en el artículo $3^{\circ}$, tal como se indicó más arriba en la nota 17 . En cualquier caso, el consenso afirma que es aquí donde se encuentra la expresión más cabal de la causa de distinción de los objetos científicos y no en el artículo $1^{\circ}$. 
intelectuales como una consecuencia del carácter ontológico de la dependencia ya indicado. En efecto, es necesario notar que el punto central que se discute en el artículo $3^{\circ}$ no reside en explicar la causa de la triple distinción de las ciencias, ni tampoco el modo de obtención o producción del objeto especulativo. Lo que se busca -más sencillamente- es responder un asunto derivado que puede resumirse en la siguiente pregunta: ¿cómo podría ser verdadera la intelección matemática al concebir separadamente de la materia lo que no existe de tal modo? ${ }^{32}$.

Siguiendo esta línea, en el corpus se afirma que, cuando el intelecto distingue, lo hace de modos diferentes según sus dos operaciones. Ahora bien, estas últimas responden a una dualidad presente en las cosas: la primera a la naturaleza del ente y la segunda a la existencia del mismo "que en los compuestos resulta de la congregación de los principios de la cosa, o es concomitante con la misma naturaleza simple de la cosa, como en las sustancias simples"33. Luego, se sigue que las distinciones que corresponden a cada una de las operaciones están ordenadas a reconocer sendos modos de separación ya sea en la naturaleza de la cosa, ya sea en su modo de existencia resultando que solo habría falsedad en el intelecto cuando este considerara separadas existencialmente dos cosas que solo presentaran una distinción en el plano de la naturaleza. En otras palabras, el intelecto no es verdadero cuando distingue según su segunda operación dos cosas que coexisten como principios de un mismo ente, pero sí es verdadero cuando las distingue según la primera operación, que consiste en concebir un principio formal sin todo aquello que no le pertenece de suyo a su naturaleza. Este modo de distinción recibe aquí propiamente el nombre de abstracción y, como es doble la relación que puede darse entre las cosas distinguidas, doble será también la abstracción.

Ahora bien, aplicando esto a la cuestión tratada, resulta que el intelecto no es falso cuando concibe los entes matemáticos sin materia sensible porque ellos no son más que formas cuantitativas que, como tales, son ontológicamente primeras a la materia sensible (abstractio formae a materia). Del mismo modo, tampoco es falso cuando concibe la esencia de un ente compuesto sin todo aquello que pertenece a tal o cual individuo porque tales particularidades no pertenecen a la ratio de la esencia ni entran en su definición (abstractio universalis a particulari). Así, desde este punto de vista, resulta claro que los tres tipos de distinción indicados no son más que el modo del intelecto de reconocer y adecuarse a los tres grados de remoción o separación que las propias cosas

32 Cf. Super De Trinitate, q. 5, a. 3, arg. 1, lin. 1-9, pp. 144-145.

33 Super De Trinitate, q. 5, a. 3, co., lin 102-105. 
presentan con respecto a la materia y que fueron introducidas en el artículo $1^{\circ}$. Es en razón de tal adecuación que el intelecto no es falso cuando conoce los objetos de la matemática independientemente de la materia: “...porque también según la cosa aquello que pertenece a la naturaleza de la línea no depende de aquello que hace que la materia sea sensible, sino más bien al revés"34.

Por lo mismo, la introducción de las operaciones intelectuales dentro del corpus del artículo no tiene como finalidad explicar causalmente la distinción de objetos sino poner en claro, frente a una objeción determinada, la tipicidad característica de la separación de lo matemático, la cual no es existencial pero aun así es real ${ }^{35}$.

Con lo dicho, Tomás da por resuelto el problema: hay verdaderamente tres tipos de ciencia especulativa porque son tres las clases de especulables según el grado de remoción de materia y movimiento. Sin embargo, el análisis de los textos muestra que, en toda su exposición, jamás se recurre a la noción de abstracción como procedimiento intelectual para fundamentar la diferenciación de los especulables entre sí y, por ende, la división de las ciencias especulativas. Antes bien, se establece que el fundamento de dicha división está puesto fundamentalmente en la realidad: porque esta es compleja en cuanto a los tipos de entes que existen -y algunos de ellos son en sí mismos complejos en tanto están compuestos por diversos géneros de principios entitativos o de formas- entonces las ciencias especulativas se dividen para poder abarcarlos a todos. Son estos entes y principios entitativos los que se distinguen entre sí por su diferente grado de remoción de la materia y el movimiento.

Valga agregar aquí que con todo esto no se quiere decir que no exista una abstracción como operación intelectual. Admitir tal cosa sería ir en contra de lo afirmado explícitamente en el corpus del artículo $3^{\circ 36}$. Más bien, se intenta mostrar que tal operación no es la causa de la distinción de los objetos como

34 Super De Trinitate, q. 5, a. 3, ad 1.

35 Cf. Gelonch, S.R.M., Separatio y objeto de la metafisica. Una interpretación textual del Super Boethium De Trinitate, q5 a3, de Santo Tomás de Aquino, pp. 254-255 donde se indica de qué modo este recurso a las operaciones del intelecto responde a la intención del Aquinate de superar las particularidades de cierto platonismo presente entre algunos de sus contemporáneos. En este sentido, Tomás logra justificar que no todo lo que el intelecto entiende separadamente debe existir separadamente en la realidad, como habría entendido Platón al verse en la necesidad de postular las ideas. Pero esto no implica admitir que la necesidad e inmaterialidad de los especulables sea fruto de la operación intelectual. Por el contrario, los mismos son las formas de las sustancias sensibles que están en los compuestos pero que no se generan ni corrompen, salvo por accidente (Cf. Super De Trinitate, q. 5, a. 2, co.).

${ }^{36}$ Y de muchos otros textos donde se define la abstracción como aquella operación intelectual que caracteriza el modo humano de conocer en estado de vía. 
se ha pretendido tradicionalmente. Por el contrario, Tomás recurre a la distinción de operaciones solo para explicar de qué modo el intelecto distingue de diversas maneras para adecuarse a los distintos modos de separación presentes en la realidad. Así, el sentido epistemológico de la abstracción no parece encontrarse en este texto simplemente porque el Aquinate no considera necesario recurrir a la causalidad de una operación intelectual para justificar la distinción de los objetos. Por ello, puede concluirse que existe entonces una inadecuación entre lo que numerosos comentadores entienden que se afirma aquí acerca de la abstracción y lo que realmente se dice acerca de ella; pero esto que se manifiesta en un texto de juventud, ¿ocurre en otros textos posteriores?

\subsection{La abstracción en la Summa Theologiae: el caso de I, q. 85, a. 1.}

La Suma de Teología contiene numerosos pasajes en los cuales se hace referencia a la abstracción. Un puñado de ellos destaca sobre el resto debido, principalmente, a la atención que han recibido por parte de diversos comentaristas. Tal es el caso, por ejemplo, de Summa Theologiae I, q. 40, a. 3, que es comentado por Cayetano ${ }^{37}$ y citado por Juan de Santo Tomás ${ }^{38}$ como uno de los lugares donde el Aquinate -más allá de no estar puntualmente refiriéndose a ningún tema específicamente relacionado con el conocimiento humano o con la ciencia especulativa- haría mención de la doctrina de la doble abstracción: total y formal ${ }^{39}$.

Con todo, en este punto resulta más interesante analizar otro de los textos, el cual ha suscitado una discusión incluso más duradera. Se trata de

37 Cf. S. Th. I, q. 40, a. 3, "Commentaria Cardenalis Caietani" (v. IV, p. 416).

38 I. a. S. Thoma. Cursus philosophicus thomisticus. "Ars Logica”, II, q. 27, a. 1, p. 822.

39 Concretamente, el artículo, situado dentro de la consideración de las Personas Trinitarias, se titula textualmente: "Si, abstraidas por el intelecto las relaciones de las personas, permanecen diferenciadas las hipóstasis". En su corpus, el Aquinate recurre a una doble abstracción del intelecto para poner de manifiesto la unidad intima entre relación e hipóstasis al probar la imposibilidad de conservar la segunda haciendo abstracción de la primera. Si esto es así, es porque la relación no es algo añadido a la hipóstasis sino, antes bien, la causa de su distinción y constitución. Si bien no es mi intención discutir en particular este texto, consideramos interesante referir al análisis que realiza Santiago Gelonch del mismo y de su recepción posterior en la tradición. En síntesis, alli indica que la consideración que hace Tomás de Aquino de la abstracción posee una naturaleza intimamente ontológica en tanto la distinción intelectual gira en torno a qué sea realmente lo conocido: si sustancia o accidente, todo o parte, etcétera, y no a qué tipo de procedimiento distinto emprenda el propio entendimiento. Así, "la diferenciación en la abstracción se da en cuanto conoce realidades ontológicamente distintas" (Gelonch, S.R.M., Separatio y objeto de la metafisica. Una interpretación textual del Super Boethium De Trinitate, q5 a3, de Santo Tomás de Aquino, p. 68) y no harian referencia a procedimientos intelectuales especiales que, como tales, dieran origen a los objetos intelectuales. 
la respuesta a la segunda objeción del artículo $1^{\circ}$ de la cuestión 85 , también ubicada en la Prima Pars. Como lo indica el título del artículo, a saber, "Si nuestro intelecto conoce abstrayendo las especies desde los fantasmas", el mismo posee como tema central la abstracción, y demuestra en su corpus que el intelecto humano, unido al cuerpo, conoce las cosas materiales mediante la abstracción a partir de los fantasmas.

Pero en la segunda objeción se presenta un problema que, por su importancia para esta investigación, conviene citarlo íntegramente: "Las realidades materiales son realidades naturales en cuya definición entra la materia. Pero nada puede ser entendido sin conocer lo que entra en su definición. Por lo tanto, los seres materiales no pueden ser entendidos sin la materia. Pero la materia es principio de individuación. Por lo tanto las realidades materiales no pueden ser entendidas abstrayendo lo universal de lo particular, en lo cual consiste abstraer las especies inteligibles de las imágenes" (S. Th. I, q. 85, a. 1, arg. 2.).

Se trata de una concatenación de dos silogismos que -tomados en conjunto- apuntan a dar por tierra con la posibilidad de que algún ente material pueda ser conocido separadamente de la materia, esto es, por abstracción desde los fantasmas. Un modo más analítico de ordenar los elementos sería el siguiente:

Primer silogismo

A) Todo ente material incluye materia en su definición

B) Ningún ente puede ser conocido sin aquello que entra en su definición

C) Ningún ente material puede ser conocido sin materia

Segundo silogismo:

D) Abstraer las especies de las imágenes implica conocer sin materia

E) Ningún ente material puede ser conocido sin materia (según C)

F) Ningún ente material puede ser conocido por abstracción desde las imágenes

Lógicamente, la proposición a vencer aquí es la formulada en el punto $\mathrm{F}$, pero, para ello, es necesario demostrar que todo el argumento no constituye más que una falacia. Tomás logra esto al indicar que el término materia, que está involucrado en ambos silogismos, es equívoco y que -por lo tanto- la conclusión C no podría ser lícitamente trasladada al segundo silogismo en carácter de premisa menor. En otras palabras, la materia que se dice estar incluida en la definición de los entes materiales (primer silogismo) no se identifica con la materia que funciona como el principio de individuación de los mismos (segundo silogismo). Esta última es la que denomina materia signada o individual y es la 
que se deja de lado con ocasión de cualquier conocimiento intelectual por ser incompatible con él. Sin embargo, el análisis de Tomás no se limita simplemente a aclarar esto, sino que procede a distinguir los distintos tipos de materia desde los cuales la inteligencia está o no posibilitada de abstraer sus especies. Este es, precisamente, el texto que ha obtenido mayor atención: "Por consiguiente, el intelecto abstrae la especie del ente natural de la materia sensible individual, pero no de la materia sensible común... Pero las especies matemáticas pueden ser abstraídas por el intelecto de la materia sensible no solo individual sino también común. Pero no de la materia inteligible común sino solo de la individual... Pero hay algunas que pueden ser abstraídas también de la materia inteligible común, como ente, uno, potencia y acto, las cuales también pueden existir sin materia como es patente en las sustancias inmateriales" (S. Th. I, q. 85, a.1, ad 2).

Es Tomás de Vio el que, en su comentario, da a entender que la enumeración del Aquinate hace referencia a los tres grados de abstracción formal del intelecto pasivo ${ }^{40} \mathrm{y}$, a partir de allí, la crítica académica ha asociado estas tres abstracciones del intelecto con las distintas estrategias especulativas orientadas a obtener los objetos científicos, se admita o no la doctrina de la abstracción formal ${ }^{41}$. Pero, como en el caso anterior, el análisis del contexto dentro del cual se encuentran insertas las líneas citadas muestra que tal interpretación

${ }^{40}$ Cf. S. Th. I, q. 85, a. 1, "Comentaria Cardenalis Caietani” (V, p. 322). Concretamente, lo que Cayetano considera que se obtiene a través de esta triple abstracción es lo que en otro lado llama "concepto objetivo". Según su visión, constituiría el quod que conoce el intelecto y sobre el que versa la ciencia. Con todo, resulta muy discutible la introducción de este elemento en tanto el mismo a) no encuentra antecedentes explícitos en la obra del Aquinate y b) parece ir en contra del propio espíritu de la letra de Tomás al poner como objeto conocido un producto del intelecto. Para más detalles sobre este asunto $C f$. Muñoz Medina, C., "Concepto formal y concepto objetivo en Cayetano. Un análisis a partir de su comentario al De Ente et Essentia”, en: Estudios Filosóficos, v. LXII, 179 (2013), pp. 49-61.

41 Incluso, como ya se ha indicado, la idea común sostenida por un buen número de académicos consiste en considerar este pasaje como paralelo a Super De Trinitate q. 5, a. 3, co., ya sea porque piensan que expresan la misma doctrina de fondo pero con distintos términos (el caso más paradigmático lo constituye M.-V. Leroy, en "Le savoir speculatif", pp. 336-338, pero también pueden citarse E.D. Simmons en el artículo "The Thomistic Doctrine of the Three Degrees of Formal Abstraction", 1959, pp. 42-43, y a R.M. Pascual Aguirre en La división de las ciencias especulativas en Santo Tomás de Aquino, p. 117), ya sea porque piensan que constituyen distintos modos de resolver un mismo problema en diferentes estadios de la vida del Aquinate (Cf. Guil Blanes, F., "Abstracción del todo, de la forma y por juicio negativo, según el comentario tomista al 'De Trinitate' de Boecio”, en: Estudios Filosóficos, V (1954), pp. 389-404, p. 393-398; Ponferrada, G.E., "Nota sobre los 'grados de la abstracción", p. 279). Por nuestra parte, consideramos que ambas posturas son erróneas en tanto comparten el equívoco fundamental de creer que en ambos textos se está discutiendo la obtención de diversos objetos especulativos mediante la operación abstractiva humana. 
no solo no es necesaria sino, incluso, problemática. En efecto, existe un puñado de motivos que alientan la sospecha de que, nuevamente, las lecturas de los comentaristas posteriores van mucho más allá de lo indicado por Tomás e, incluso, que distorsionan sus conclusiones.

En primer lugar, conviene preguntarse si acaso se justifica introducir, con ocasión de la respuesta a la segunda objeción, una abstracción del intelecto pasivo. Esta cuestión es relevante toda vez que desde el título del artículo y a lo largo de todo el cuerpo queda claro que Tomás hace referencia a la abstracción de las especies intelectuales desde los fantasmas, operación que -allende lo que aquí se afirma- es atribuida explícitamente al intelecto agente en un amplísimo número de pasajes ${ }^{42}$. De esta manera no parece haber fundamentos suficientes desde lo textual que permitan hablar en la respuesta a la objeción segunda de una operación abstractiva distinta y adicional a la propia del intelecto agente. Por el contrario, lo que allí se sigue discutiendo es el mismo proceso de obtención de las especies inteligibles con el único agregado de la ya citada aclaración con respecto a qué tipo de materia es la que conserva y la que se suprime en el caso de cada especie.

También se impone una segunda constatación que apunta a mostrar una incompatibilidad de la indicada interpretación epistemológica del pasaje ya no en el plano terminológico sino en el conceptual: cuando Tomás habla de estas tres especies, en ningún momento las presenta como los objetos respectivos de las tres ciencias especulativas. Esto queda claramente demostrado por dos motivos de gran importancia. Primero, porque el tercer tipo de especie -que incluye ente, uno y acto- no podría corresponderse completamente con el objeto de la metafisica, ciencia que también (y sobre todo, podría agregarse) trata sobre las sustancias separadas, las cuales no pueden bajo ningún aspecto ser abstraídas desde la materia porque son completamente inmateriales ${ }^{43}$. Segundo, porque en el artículo segundo de la propia cuestión 85 el Aquinate declara explícitamente que las especies abstraídas por el intelecto no pueden constituirse como sus

\footnotetext{
42 Se destacan, entre otros numerosísimos casos: Super Sent., lib. 4, d. 50, De veritate, q. 2, a. 6, ad 1, Q. d. de anima, a. 4, ad 9 y a. 5, s.c. 2, Contra Gentiles, lib. 2, cap. 75 , n. 8 y cap. 77 n. $3, S$. Th. I, q. 79 , a. 4 , ad 4.

$43 C f$. en este sentido el carácter de inseparabilis que les atribuye en su exposición sobre el capítulo segundo del De Trinitate queriendo indicar, mediante un giro poco menos que sorprendente, que tales objetos no pueden ser separados de la materia porque ya existen fuera de ella. También resulta interesante recordar lo que en su momento indicó Merlan en su respuesta a las pretensiones de Leroy de querer ver aquí la enumeración completa de los objetos de ciencia especulativa (Cf. Leroy, M-V., "Le savoir speculatif", pp. 336-338, y Merlan, P., "Abstraction and Metaphysics in St. Thomas' Summa", p. 287).
} 
objetos cognoscitivos. Si así fuese, a) toda ciencia versaría únicamente sobre ideas en la mente y no sobre la realidad, reduciéndose forzosamente a la lógica; y b) no existiría la posibilidad de que algún juicio humano fuese falso en tanto resultaría imposible que la inteligencia no se adecue a sus propias especies ${ }^{44}$. Consecuentemente, las especies enumeradas en el artículo $1^{\circ}$ no son el quod del conocimiento (es decir, lo que se conoce) sino el qua, o el medio a través del cual el hombre conoce en estado de via ${ }^{45}$. Si esto es así, debe admitirse entonces que ni las especies pueden ser los objetos de la ciencia, ni la abstracción que les da origen puede tener un rol fundamentativo con respecto a la división de las ciencias especulativas ${ }^{46}$.

Finalmente, existe un tercer elemento que evidencia la inadecuación entre lo que afirma Tomás aquí y la hermenéutica que se ha impuesto y, aunque podría pensarse que se trata de una precisión demasiado sutil, no por ello deja de ser totalmente relevante. En efecto, debe notarse que en ningún momento de la respuesta a la segunda objeción se indica que la distinción entre las tres especies intelectuales se fundamente en última instancia en la actividad abstractiva que realiza el intelecto humano. Por el contrario, en el mismo texto Tomás reconoce que hay una causa ulterior que es la que permite o no que el intelecto pueda abstraer una especie de tal o cual materia: "Así como [el intelecto] abstrae la especie de hombre de esta carne y estos huesos, que no pertenecen a la razón de la especie sino que son partes del individuo, como se afirma en el libro VII de la Metafisica, y por este motivo puede ser considerada sin ellos. Pero la especie del hombre no puede ser abstraída por el intelecto de las carnes y los huesos" (S. Th. I, q. 85, a. 1, ad 2).

Lo aquí dicho se explica si se tiene en cuenta que, ante todo, la especie que el intelecto agente abstrae desde los fantasmas no es más que una semejanza de la esencia de la cosa extramental. Tal esencia o quididad es precisamente lo que se conoce a través de la especie abstraída cuando esta última es recibida por el intelecto pasivo. Ahora bien, si la especie intelectual puede cumplir este rol es porque la misma representa con fidelidad dicha esencia, conteniendo todo aquello que pertenece a esta y dejando de lado aquello que no le pertenece. Esto significa que, si el intelecto puede abstraer las especies naturales de la materia signada, las especies matemáticas de la materia sensible y las especies

\footnotetext{
44 Cf. S. Th. I, q. 85, a. 2, co.

45 Cf. S. Th. I, q. 85, a. 2, s.c.

46 Esta misma distinción entre lo que se conoce y aquello a través de lo cual el hombre conoce puede encontrarse en Q. d. de anima, a. 2 ad 5.
} 
metafísicas de toda materia, es porque las esencias de las cuales cada una de ellas son semejanzas ya presentan de antemano una distinción con respecto a cada uno de dichos tipos de materia. De este modo, la distinción entre los tres tipos de especie en el texto de Santo Tomás no se explica causalmente por una diferente estrategia considerativa aplicada por el intelecto humano sino por su relación con la materia; relación que es, en sí misma, constitutiva de las rationes de las especies como tales en tanto semejanzas de una determinada esencia ${ }^{47}$. A esto apunta la referencia al libro VII de la Metafísica, donde se explica la distinción entre aquello que pertenece a la esencia de una cosa y aquello que pertenece al individuo existente como tal ${ }^{48}$, y también la aceptación por parte de Tomás de una de las premisas de la objeción 2: que si la esencia de cualquier ente natural posee materia (sensible, en este caso), entonces la especie de la primera no puede ser abstraída de la segunda.

En sintesis, el análisis de este texto permite concluir que-contrariamente a la opinión mayoritaria en el campo del tomismo- no existe aquí una referencia a una operación abstractiva científica llevada a cabo por el intelecto pasivo. Más bien, la abstracción descrita se identifica con la actividad del intelecto agente que resulta clave para que se perfeccione el conocimiento humano en estado de vía. Por ende, tampoco puede decirse que dicho procedimiento tenga una relevancia causal en cuanto a la determinación de los objetos científicos y a la división de los propios hábitos, ya que el mismo a) está ordenado solo a la obtención de los instrumentos mediante los cuales conoce el hombre (especies abstractas) y no de los objetos que conoce; y b) es dependiente de la constitución que de suyo presentan las esencias de las cosas conocidas.

\section{Corolario}

Recapitulando los resultados del análisis realizado en las páginas anteriores corresponde aclarar nuevamente que con lo dicho no se pretende negar que haya en Tomás de Aquino una abstracción del intelecto. Tal cosa queda probada ampliamente por los propios textos citados. Ahora bien, lo que sí consideramos necesario rechazar es la idea de que la operación intelectual

\footnotetext{
47 Cf. Gelonch, S.R.N., Separatio y objeto de la metafisica. Una interpretación textual del Super Boethium De Trinitate, q5 a3, de Santo Tomás de Aquino, p. 69. Esta opinión resulta compatible con las conclusiones a las que arriba Therese Scarpelli Cory en los artículos citados más arriba en lo que refiere al modo en que debe ser entendida la abstracción del agente en Tomás de Aquino: no como la producción selectiva de la especie, sino como la actualización de la inteligibilidad potencial que posee el fantasma en tanto semejanza de un ente que posee formas (Cf. supra, p. 4).

${ }^{48}$ Cf. In Metaphysic., VII, lectio 10.
} 
que se describe tanto en el Super De Trinitate q. 5 como en Summa Theologiae I, q. 85, a. 1, ad 2 se identifique con una abstracción científica llevada a cabo por el intelecto pasivo que tenga como propósito garantizar el acceso al plano de lo objetivo y fundamentar así la distinción de las ciencias.

Adoptar esta óptica implica no solo ir más allá de lo que dicen los textos, sino -más grave aun- forzar su sentido. Ni siquiera podría concluirse legítimamente que ambos constituyan pasajes paralelos, ya que mientras el Super De Trinitate tiene como tema central la cuestión de la división de las ciencias especulativas, el texto de la Summa gira en torno a la explicación de una de las limitaciones particulares del conocimiento humano en tanto se trata del acto de un compuesto de alma y cuerpo: la mediación de las especies abstraídas desde el fantasma imaginativo. Así, pretender que ambos textos tratan de lo mismo equivale a decir que para el Aquinate el conocimiento científico como tal se identifica sin más con el conocimiento humano ${ }^{49}$.

Con todo, sí es posible detectar ciertos puntos de contacto entre ambos textos, y uno de ellos compete directamente a la abstracción. Efectivamente, en ambos casos el término designa en líneas generales una distinción del intelecto mediante la cual se considera algo mientras se deja de lado otra cosa. También coinciden en diferenciar tipos de abstracción según sea aquello que se considera y aquello que se deja de lado. Pero -lo que es más importante- en ambos textos se deja muy en claro que la causa fundamentativa de tales distinciones intelectuales reside en la propia realidad que es considerada y su tipicidad ontológica: si es simple o compuesta, si es sustancia o accidente, si su esencia demanda la coparticipación de tal o cual tipo de materia, etcétera. Por ende, la causa de la distinción de los objetos reside en la propia complejidad de lo real y no en el modo de considerar o en la estrategia cognoscitiva. Por lo demás, el intelecto hace siempre lo mismo: considerar lo actual o formal sin lo potencial o material.

En sintesis, lo que el análisis precedente muestra es que existe una diferencia de planos dentro de los cuales Tomás y sus comentadores (tanto clásicos como contemporáneos) hablan acerca de la abstracción. Mientras que para los últimos dicha operación tiene como finalidad vincular dos órdenes

\footnotetext{
49 Incluso, en tanto esta mirada del asunto permite distinguir claramente los contextos de cada uno de estos pasajes, la diferencia terminológica entre ambos (esto es, el uso de separación en uno y de abstracción en otro) no causa ningún problema y estaría lejos de poder ser considerada un caso de asignación indiscriminada como lo califican Simmons o Vicente Burgoa (Cf. Simmons, D., "The Thomistic Doctrine of the Three Degrees of Formal Abstraction", pp. 44-45 y Burgoa, V., “¿Abstracción formal o separación? Desde Tomás de Aquino a Cayetano”, p. 247).
} 
diversos (el de las cosas que existen y el de la inteligencia que las conoce) ${ }^{50}$, nada hay en los textos del primero (o por lo menos en los analizados) que impida pensar que la distinción de objetos científicos no es otra cosa que una distinción entre esencias, que se basa o bien en la simplicidad de las mismas o bien en el tipo de composición que presentan con el sustrato que requieren para ser entendidas ${ }^{51}$. Luego, dentro de este contexto, la abstracción vista como una operación intelectual es posterior a y dependiente de dicha diversidad de esencias en tanto se refiere al modo de adecuarse del intelecto con respecto a la realidad (tal como se describe en Super De Trinitate q. 5, a. 3). En el caso particular del hombre en estado de vía, tal adecuación asume la modalidad de una producción de especies inteligibles merced a la luz del intelecto agente para su posterior recepción en el pasivo (que es lo que se plantea en $S$. Th. q. 85, a. 1).

Pues bien, siendo esto así, consideramos necesario revisar con mayor detenimiento el modo en que se ha entendido tanto la abstracción como la división de las ciencias según el pensamiento de Tomás, volviendo a la letra de sus textos y obviando (al menos en un primer momento metodológico) las derivaciones que han tenido sus afirmaciones en los escritos de sus comentaristas. Después de todo, en este caso como en otros, el pensamiento de Tomás de Aquino se entiende mejor por lo que le precede que por lo que le sigue ${ }^{52}$.

Recibido: 08/02/2017

Aceptado: 01/03/2018

\footnotetext{
50 "El supuesto inicial del que parte la abstracción formal es que el intelecto y la cosa pertenecen a dos órdenes diferentes. Nos encontramos ya en un universo psicologizado, en que sujeto y objeto han perdido parte de la unidad común que poseían en Aristóteles, y donde el intelecto se caracteriza por su virtud formalizadora, y sus facultades se han transformado en estructuras formales que aún no son enteramente a priori pero que tampoco viven ya al unísono con las cosas del mundo natural 'exterior"' (Cf. León Florido, F., "Prólogo" en: Muñoz Medina, C., Ciencia y objetividad en Cayetano, Santiago de Chile: RIL, 2016, p. 16). Aunque tales cosas sean dichas de la abstracción formal y la misma sea explícitamente rechazada por un buen número de los intérpretes contemporáneos de Tomás, considero, sin embargo, que la disociación denunciada también constituye el contexto de todo planteo que introduce una abstracción científica. Solo así tiene sentido la necesidad de postular diferentes estrategias cognoscitivas que permitan distinguir los objetos.

${ }^{51} \mathrm{Si}$ bien su análisis requeriría de un trabajo adicional, puede adelantarse aquí que textos clave como el Proemio del In Metaphysica o el comienzo del In De sensu et sensato permiten arribar a la misma conclusión.

${ }^{52}$ Cf. Reindhart, E., "Conversación en Fribourg con Jean-Pierre Torrell", en: Anuario de Historia de la Iglesia, 15 (2006), pp. 305-332, p. 309.
} 


\section{Bibliografia}

Acerbi, A, “Aquinas's Commentary on Boethius' De Trinitate” en: Review of Metaphysics, v. LXVI, n. 2 (2012), pp. 317-338.

Aquinatis, T., Commentaria in octo libros Physicorum Aristotelis, Opera omnia iussu impensaque Leonis XIII. P. M. edita. vol. II, Ex Typographia Polyglotta S. C. de Propaganda Fide, Roma, 1884.

Summa Theologiae. Pars Prima. Opera omnia iussu impensaque Leonis XIII P. M. edita. v. IV, Ex Typographia Polyglotta S. C. de Propaganda Fide, Romae, 1888. Pars prima Summae theologiae. Opera omnia iussu impensaque Leonis XIII P. M. edita. v. V, Ex Typographia Polyglotta S. C. de Propaganda Fide, Roma, 1889. In duodecim libros Metaphysicorum Aristotelis expositio. ed. por M. R. Cathala; R. M. Spiazzi, $2^{\circ}$ Marietti, Taurini-Romae, 1971.

- De ente et essentia. Opera omnia iussu Leonis XIII P. M. edita. v. LXIII, pp. 315381 Editori di San Tommaso, Roma, 1976.

Super Boethium De Trinitate. Opera omnia iussu Leonis XIII P. M. edita. Vol. 50, pp. 1-230 Commissio Leonina-Éditions Du Cerf, Roma-Paris, 1992.

Aristóteles, Metafisica, Calvo Martínez, T. (trad.), Gredos, Madrid, 2007.

Bonino, S-T., "Le thomisme de 1962 à 2012. Vue panoramique", en: Nova et Vetera 87 (2012), pp. 419-446.

Cory, T.S., "Averroes and Aquinas on the Agent Intellect's Causation of the Intelligible" en: Recherches de Théologie et Philosophie Médiévales, v. LXXXII, n. 1 (2015), pp. 1-60. "Rethinking Abstractionism: Aquinas's Intellectual Light and Some Arabic Sources" en: Journal of the History of Philosophy, v. LIII, n. 4 (2015), pp. 607-646. https:// doi.org/10.1353/hph.2015.0074

Elders, L, Faith and Science: an Introduction to St. Thomas' Expositio in Boethii De Trinitate, Studia Universitatis S Thomae in Urbe. Herder, Roma, 1974.

Ferrari, L.C., "A Thomistic Appraisal of Thomas de Vio Cajetan's 'Abstractio Totalis", en: Angelicum: Periodicum Trimestre Pontificiae Studiorum Universitatis e Sancto Thoma Aquinate in Urbe 42 (1965), pp. 441-462.

Geiger, L-B., "Abstraction et Séparation d' après Saint Thomas In de Trinitate, Q. 5, A. 3", en: Penser avec Thomas d' Aquin, Ruedi I. (ed.), Friburgo/París: Éditions Universitaires-Éditions du Cerf, 2000, pp. 139-183.

Gelonch, S.R.M., Separatio y objeto de la metafisica. Una interpretación textual del Super Boethium De Trinitate, q5 a3, de Santo Tomás de Aquino, Colección de pensamiento medieval y renacentista, Cruz Cruz, J. (ed.), Pamplona: EUNSA, 2002.

Guil Blanes, F., "Abstracción del todo, de la forma y por juicio negativo, según el comentario tomista al 'De Trinitate' de Boecio”, en: Estudios Filosoficos V (1954), pp. 389-404.

Kerr, F., “¿Un tomismo analítico?”, en: Civilizar Ciencias Sociales y Humanas 12 (2012), pp. 149-158. https://doi.org/10.22518/16578953.108

Kockelmans, J.J., The Metaphysics of Aquinas: a Systematic Presentation, Lovaina: Bibliotheek van de Faculteit Godgeleerdheid, 2001. 
Leroy, M-V., "Le savoir speculatif", en: Revue Thomiste: Revue doctrinale de Theologie et de Philosophie, v. XLVIII, n. 1-2 (1948), pp. 236-339.

López Salgado, C., "Abstractio y separatio como acceso a la metafísica”, en: Sapientia v. XIX, n. 72 (1964), pp. 102-116.

Llano, C., "La 'Introducción' a la Crítica de la Razón Pura y el Comentario al De Trinitate de Boecio de Santo Tomás de Aquino. (Spanish)”, en: Tópicos. Revista de Filosofía, n. 21 (2001), pp. 27-67. https://doi.org/10.21555/top.v21i1.315

MacIntyre, A.C., First Principles, Final Ends, and Contemporary Philosophical Issues. The Aquinas Lecture, Milwaukee: Marquette University Press, 1990. https://doi. org/10.1017/cbo9780511819797.009

Mansion, S., 'L' intelligibilité metaphysique d'apres le 'Proemium' du Commentaire de Saint Thomas a la 'Metaphysique' d'Aristote”, en: Rivista di Filosofia Neo-Scolastica 70 (1978), pp. 49-62.

Maritain, J., Distinguer pour unir, París: Desclée, 1932.

Los grados del saber. Distinguir para unir, Buenos Aires: Club de Lectores, 1978.

Maurer, A.A., The Division and Methods of the Sciences. Mediaeval Sources in Translation; Turnhout: Brepols, 1986.

Mendoza, J.M.F., "La epistemología tomista: un designio de la originaria noción tomística de ciencia”, en: Ciencia Tomista, v. CXL, n. 452 (2013), pp. 389-416.

Merlan, P., "Abstraction and Metaphysics in St. Thomas' Summa", en: Journal of the History of Ideas, 14 (1953), pp. 284-291. https://doi.org/10.2307/2707477

_ From Platonism to Neoplatonism, v. III, La Haya: Martinus Nijhoff, 1975. https:/ / doi.org/10.1007/978-94-010-1592-9

Moya Cañas, P., "Inmanencia, intencionalidad y representación en Tomás de Aquino", en: Veritas: Revista de Filosofia, n. 28 (2013), pp. 113-131. https:/ / doi.org/ 10.4067/ s0718-92732013000100005

Muñoz Medina, C.P.D., "Concepto formal y concepto objetivo en Cayetano. Un análisis a partir de su comentario al De Ente et Essentia”, en: Estudios Filosóficos, v. LXII, n. 179 (2013), pp. 49-61.

"Notas sobre el primum cognitum en Cayetano y su vínculo con la metafísica", en: Anales del seminario de Historia de la Filosofia 32 (2015), pp. 127-142. https:// doi.org/10.5209/rev_ashf.2015.v32.n1.48682

Ciencia y objetividad en Cayetano, Santiago de Chile: Ril, 2016.

Nordberg, K., "Abstraction and Separation in the Light of the Historical Roots of Thomas' Tripartition of the Theoretical Sciences", en: Knowledge and the Sciences in Medieval Philosophy. Proceedings of the Eighth International Congress of Medieval Philosophy (1987), Tyorinoja, R. (ed.), Helsinski: Ylipostipaino, 1990, pp. 144-159.

Nuñez Pliego, J.M., Abstracción y separación. Estudio sobre la metafísica de Tomás de Aquino, en: Cuadernos de Anuario Filosófico, González, A. (ed.), Pamplona: EUNSA, 2008.

Pascual Aguirre, R.M. La división de las ciencias especulativas en Santo Tomás de Aquino. Excerpta ex dissertatione ad Doctorandum in Facultate Philosophiae, Pontificia Universitas Gregoriana, 2003. 
Pasnau, R., Theories of Cognition in the Later Middle Ages, Cambridge University Press, 1997.

Ponferrada, G.E., "Nota sobre los 'grados de la abstracción”, en: Sapientia 33 (1978), pp. 267-284.

Ramírez, S.M., De ipsa philosophia in universum. Edición de las obras completas de Santiago Ramírez, O. P. 2 vols. Instituto de Filosofia "Luis Vives", Madrid, 1970.

Reindhart, E., "Conversación en Fribourg con Jean-Pierre Torrell”, en: Anuario de Historia de la Iglesia 15 (2006), pp. 305-332.

Simmons, E.D. "The Thomistic Doctrine of the Three Degrees of Formal Abstraction", en: Thomist: A Speculative Quarterly Review 22 (1959), pp. 37-67. https://doi. org/10.1353/tho. 1959.0002

Thoma, Ioannis a Sancto. Cursus Philosophicus Thomisticus. Laurentius, A. (ed.), Lugduni, 1678.

. Cursus philosophicus thomisticus. Reiser, B. (ed.), v. I, "Ars Logica”, Marietti, Turin, 1930.

Vicente Burgoa, L., “¿Abstracción formal o separación? Desde Tomás de Aquino a Cayetano”, en: Estudios Filosóficos 138 (1999), pp. 211-256.

"La abstracción formal y la validación del razonamiento inductivo. (Spanish)", en: Sapientia 62 (2007), pp. 129-178. 